\title{
Robust and efficient numerical methods for the optimal control of spatially distributed biological systems
}

\author{
Carlos Vilas, Eva Balsa-Canto*, Julio R. Banga and Antonio A. Alonso
}

\begin{abstract}
In silico experimentation has opened new ways to analyze biological systems behavior under different conditions. The incorporation of an outer optimization loop may help to find the right operation conditions to achieve specific goals (maximization of a given product concentration, minimization of process energy/time, etc.). Mathematically, this is stated as a dynamic optimization problem being particularly challenging when the system is described by nonlinear sets of partial differential equations as well as when constraints are considered. These issues impose a number of difficulties, such as the presence of suboptimal solutions, which call for robust and efficient numerical techniques.

In this work, the control vector parametrization approach is combined with reduced order methods and suitable hybrid global optimization methods to overcome such difficulties. The capabilities of this strategy are illustrated considering the solution of two challenging problems: bacterial chemotaxis and the FitzHugh-Nagumo model. The presented methodology can be used for the efficient dynamic optimization of generic distributed biological systems.
\end{abstract}

\section{INTRODUCTION}

In recent years modeling of biological system has received considerable attention. Nevertheless, only in few occasions those models have been used to design or to optimize desired biological behaviors. This may be explained by the difficulty on formulating and solving those problems but also in the limited number of software tools that may be used for that purpose. In this regard, the recently developed toolbox DOTcvpSB [10] can handle the dynamic optimization of lumped systems (described in terms of ordinary differential equations), such as those related to biochemical processes [3], or to biomedical systems [18]. However, many biological systems of interest are being modelled by sets of partial differential equations (PDE). This is particularly the case of reaction diffusion waves in biology or spatial organization in cell signaling. The scarce works related to the optimization of this type of systems [11], [12] reveal that the problem presents significant computational and conceptual challenges due mainly to the presence of suboptimal solutions and to the computational cost associated to the simulation and, thus, the optimization.

Global optimization techniques capable of dealing with multimodal problems can be used to find the global solution. Unfortunately, with these techniques, the computational cost increases exponentially with the number of decision variables. This aspect is particularly critical for PDE systems as they are usually solved with spatial discretization techniques

This work was not supported by any organization

(Bio)Process Engineering Group, IIM-CSIC, Eduardo Cabello, 6, 36208 Vigo, Spain. ebalsadim.csic.es and the result is a large scale dynamic system whose simulation may take from several seconds to hours. In this concern, surrogate models has been proposed as an alternative to reduce computation times. Different techniques based on kriging or radial basis functions have been incorporated to global optimization solvers [6]. However these methodologies do not integrate any knowledge about the system being optimized. For instance, radial basis functions are based on nonlinear approximations of the objective function. Alternatives for PDE systems rely on the application of reduced order modeling techniques which make use of the system dynamics and try to find a low dimensional subspace which approximates such dynamics to take into account only the phenomena of interest. In particular the use of the proper orthogonal decomposition (POD) approach has demonstrated to be an excellent candidate for simulation, optimization and control [20].

This work presents the application of hybrid optimization techniques for the solution of complex dynamic optimization problems related to biological applications. Particular emphasis is paid to the efficiency and robustness of the proposed methodologies. In this regard, the use of a hybrid global-local methodology together with a control refining technique is proposed. In addition, the proper orthogonal decomposition technique will be employed when required to reduce the the computational effort of the original distributed models. To illustrate the usage and advantages of the proposed techniques two challenging case studies will be considered, namely, bacterial chemotaxis [11] and the FitzHugh-Nagumo (FHN) model [7], [14].

\section{GENERAL MODEL DESCRIPTION AND SIMULATION}

Most of biological systems can be described by coupled sets of partial and ordinary differential equations (PDEs) of the form:

$$
\begin{gathered}
\frac{\partial x}{\partial t}=\nabla \cdot(k \nabla x)-\nabla \cdot(\mathbf{v} x)+f(x, y, u) \\
\frac{\mathrm{d} y}{\mathrm{~d} t}=g(x, y, u)
\end{gathered}
$$

where $u(t)$ represent the vector of control variables. The state variables are split into spatially distributed $x(\xi, t)$ and lumped $y(t)$ variables. $f(x, y, u)$ and $g(x, y, u)$ are two given nonlinear functions which may represent for instance chemical reactions. This system must be completed with appropriate initial and boundary conditions.

The spatially distributed and nonlinear nature of biological systems of the form (1)-(2) make finding the analytical solution rather complicated, if not impossible calling for efficient 
numerical techniques. Classical numerical approaches like the finite element method (FEM) [15] or the finite differences method (FDM) [16] are based on the discretization of the spatial domain into a (usually large) number $N$ of smaller subdomains. After some algebra, the original PDE is approximated by a number $N$ of ODEs. Both methods have been successfully applied in the context of dynamic optimization [11], [2]. However in many biological models, especially those in $2 \mathrm{D}$ and $3 \mathrm{D}$, the number of discretization points $(N)$ to obtain an accurate solution might be too large for their application in optimization. Reduced order models have emerged as efficient alternatives to overcome this limitation.

Probably the most efficient order reduction technique is the proper orthogonal decomposition (POD) [17] and because of this, it will be chosen in this work to obtain the reduced order models. In this technique, the solution of the PDE system (1) is approximated by a truncated Fourier series of the form:

$$
x(\xi, t) \approx \sum_{i=1}^{N} m_{i}(t) \phi_{i}(\xi)
$$

Instead of computing directly $x(\xi, t)$ we proceed in two steps: A) computation of the basis functions $\phi_{i}(\xi)$ and B) computation of the time coefficients $m_{i}(t)$.

\section{A. The basis functions}

Basis functions are computed off-line as the solution of the following integral eigenvalue problem [17], [1]:

$$
\int_{\mathbb{V}} R\left(\xi, \xi^{\prime}\right) \phi_{i}\left(\xi^{\prime}\right) \mathrm{d} \xi^{\prime}=\lambda_{i} \phi_{i}(\xi)
$$

where $\lambda_{i}$ corresponds with the eigenvalue associated with each global eigenfunction $\phi_{i}$ and the kernel $R\left(\xi, \xi^{\prime}\right)$ is the following two point spatial correlation function:

$$
R\left(\xi, \xi^{\prime}\right)=\frac{1}{\ell} \sum_{j=1}^{\ell} x\left(\xi, t_{j}\right) x\left(\xi^{\prime}, t_{j}\right) .
$$

with $x\left(\xi, t_{j}\right)$ denoting field measurements at each instant $t_{j}$ and the summation extends over a sufficiently rich collection of uncorrelated snapshots at $j=1, \cdots, \ell$ [17]. Field measurements can be obtained either by numerical simulation or by physical experimentation. It is important to highlight that the basis functions obtained from (4) are orthogonal.

\section{B. The time dependent coefficients}

In order to compute the time dependent coefficients in Eqn (3), the original PDE system (1) is projected onto each element of the POD basis set. In this work, such projection is carried out by multiplying the original PDE by each $\phi_{i}$ and integrating the result over the spatial domain. This will lead to a ODE system of the form:

$$
\frac{\mathrm{d} m_{\mathrm{A}}}{\mathrm{d} t}=P_{\mathrm{A}} m_{\mathrm{A}}+F_{\mathrm{A}}
$$

where $m_{\mathrm{A}}=\left[m_{1}, m_{2}, \cdots, m_{N}\right]^{T}, P_{A}$ is a matrix of the form $P_{A}=\int_{\mathbb{V}} \phi_{A}(\nabla \cdot(k \nabla)-\nabla \cdot \mathbf{v}) \phi_{\mathrm{A}} d \xi$ with $\phi_{\mathrm{A}}=$ $\left[\phi_{1}, \phi_{2}, \cdots, \phi_{N}\right]$ while $F_{\mathrm{A}}$ represents the projection of the nonlinear term $F_{A}=\int_{\mathbb{V}} \phi_{A} f d \xi$.
Since, at this point, both $\phi_{A}$ and $m_{\mathrm{A}}$ are known, the solution $x$ can be recovered by applying Eqn (3). The number of elements $N$ in the basis subset $\phi_{\mathrm{A}}$ can be increased to approximate the state $x$ with an arbitrary degree of accuracy.

\section{DYNAMIC OPTIMIZATION PROBLEM FORMULATION}

Dynamic optimization, also called open loop optimal control (OCP), considers the computation of a set of timedependent operating conditions (controls) which optimize a certain performance index $J$ subject to a set of constraints. Mathematically this is formulated as follows: find $u(t)$ along $t \in\left[t_{0}, t_{f}\right]$ to minimize (or maximize) the performance index:

$$
J=\varphi\left(x\left(\xi, t_{f}\right), y\left(t_{f}\right)\right)+\int_{t_{0}}^{t_{f}} L(x(\xi, t), y(t), u(t))
$$

where $\varphi$ and $L$ are given (possibly nonlinear) functions. The set of constraints may be classified in three main groups: i) the system dynamics (1)-(2), ii) the bounds for the control variables $u^{L} \leq u(t) \leqslant u^{U}$, and iii) other constraints, which must be satisfied over the entire process time (path constraints) or at specific times (point constraints). These constraints can be expressed as: $c(x(\xi, t), y(t), u(t)) \leqslant 0$, $c\left(x\left(\xi, t_{k}\right), y\left(t_{k}\right), u\left(t_{k}\right)\right) \leqslant 0$ where $t_{k}$ is a time point.

\section{A. Dynamic optimization methods}

There are several alternatives for the solution of dynamic optimization problems from which the direct methods are the most widely used. These methods transform the original problem into a non-linear programming (NLP) problem by means of the complete parametrization ( $\mathrm{CP},[4])$, the multiple shooting (MS, [5]) or the control vector parametrization (CVP, [19]). Basically, all of them are based on the use of some type of discretization and approximation of either the control variables or both the control and state variables. The three alternatives basically differ in: the resulting number of decision variables, the presence or absence of parametrization related constraints and the necessity of using an initial value problem solver. In this work we will employ the CVP approach since it allows handling large-scale DO problems without solving very large NLPs and without dealing with extra junction constraints [2]. The CVP proceeds dividing the process duration into a number of elements and approximating the control functions typically using low order polynomials whose coefficients become the new decision variables. It is important to note that the larger the number of elements to approximate the control function, the larger the number of decision variables and the larger the computational time to find the optimum. This is particularly relevant when several control inputs are considered. However, considering a small number of control elements could result into a poor approximation to the optimal control input.

\section{B. Nonlinear programming methods}

Nonlinear programming methods may be largely classified in two groups: local and global methods. Local methods use some type of pattern search or gradient and Hessian information to find a local optimum. Unfortunately, when 
considering nonlinear distributed systems, the application of CVP usually results into a problem with multiple local optima (multimodal) [3] in which local optimization techniques will be probably trapped. In order to surmount these difficulties, global optimization methods must be used.

The different global strategies may be classified in two major groups: deterministic and stochastic. Global deterministic methods [8] use the problem's structure and guarantee global convergence for some particular problems that verify specific smoothness and differentiability conditions. However, the necessary conditions for these methods to be applicable may not be guaranteed for the cases of interest and the computational cost may become prohibitive. On the other hand, stochastic methods do not require any assumptions about the problem's structure. They make use of pseudorandom sequences to determine search directions toward the global optimum. The main advantage of these methods is that, in practice, they rapidly arrive to the proximity of the solution although convergence may not be guaranteed. Among the different classes of stochastic methods, the metaheuristics have proved to be very efficient. Although many stochastic methods can locate the vicinity of global solutions very rapidly, the computational cost associated to the refinement of the solution is usually very large. In order to surmount this difficulty, hybrid methods which combine global stochastic methods with local gradient based methods have been recently developed [6].

It must be noted that even hybrid methods may become prohibitive with an increasing number of decision variables. A possible way to deal with this problem is to use a control refining technique. This technique consists of performing successive re-optimizations with increasing control discretization level. This methodology proceeds in three steps: i) the problem is solved using a coarse control discretization with a hybrid optimization technique; ii) the control discretization level of best solution found is increased and the result is employed as starting point for a local deterministic optimization method; iii) step ii) is repeated till the established number of refinements has been achieved.

\section{Results AND Discussion}

To illustrate the usage and advantages of the proposed techniques two challenging case studies will be considered, namely, bacterial chemotaxis and the FitzHugh-Nagumo (FHN) model. The objective in both cases will be to compute the optimal control profiles which originate given desired patterns in the state variables.

\section{A. Case Study I: Bacterial chemotaxis}

Some types of cells are able to sense the presence of chemical signals (chemoattractants) and guide their movement in the direction of the concentration gradient of these signals. This process, called chemotaxis, has a role in diverse functions such as the sourcing of nutrients by prokaryotes, the formation of multicellular structures, tumor growth, etc.

The mathematical model of the chemotaxis process consists of a coupled system of PDEs describing the bacteria and chemoattractant behavior. In its $1 \mathrm{D}$ version, such model reads as follows [12]:

$$
\begin{gathered}
\frac{\partial z}{\partial t}=D \frac{\partial^{2} z}{\partial \xi^{2}}-\mu \frac{\partial}{\partial \xi}\left(\frac{z}{(1+c)^{2}} \frac{\partial c}{\partial \xi}\right) \\
\frac{\partial c}{\partial t}=\frac{\partial^{2} c}{\partial \xi^{2}}+\frac{z^{2}}{1+z^{2}}
\end{gathered}
$$

with boundary and initial conditions of the form:

$$
\begin{gathered}
\left.\frac{\partial z}{\partial \xi}\right|_{\xi=0}=\left.\frac{\partial z}{\partial \xi}\right|_{\xi=L}=\left.\frac{\partial c}{\partial \xi}\right|_{\xi=0}=0 ; \\
\frac{\partial c}{\partial \xi}=c+\left.u(t)\right|_{\xi=L} \\
z(\xi, 0)=1 ; \quad c(\xi, 0)=0
\end{gathered}
$$

where $z(\xi, t)$ and $c(\xi, t)$ represent the cell density and the concentration of the chemoattractant, respectively. $u(t)$ is the control variable. The model parameters are set to $D=0.33$, $\mu=80$ and $L=1$.

The objective is to externally manipulate the system so as to achieve a particular cell distribution. Lebiedz and coworkers [11], [12] consider several desired cell distributions among which the most difficult to achieve correspond to a Gaussian $z_{T 1}(\xi)=2.2\left(\exp \left(-25(\xi-0.5)^{2}\right)+0.1\right)$ and a constant $z_{T 2}(\xi)=1$ profile.

The optimal control problem may be then formulated as follows: Find $u(t)$ within the interval $t \in[0,1]$ so as to minimize the deviation of the cell density as compared to the desired spatial distribution. Mathematically, this is:

$$
\min _{u} J_{k} ; \quad \text { with } \quad J_{k}=\frac{1}{2 n_{\xi}} \sum_{j=1}^{n_{\xi}}\left(z_{j}\left(t_{f}\right)-z_{T, k j}\right)^{2}
$$

where the subindex $j$ regards a particular spatial position while $k=1,2$ represents the Gaussian and constant profiles, respectively. Optimal control problem (12) is subject to:

- The system dynamics described by Equations (8)-(11)

- Bounds on the control variable $0 \leqslant u(t) \leqslant 1$.

The sub-cases will be referred to as OCP1 (for the Gaussian distribution) and OCP2 (for the constant profile).

1) Results: The finite differences method is employed in this case study to numerically compute the solution of system (8)- (11). In order to avoid spurious numerical solutions, a comparison among different schemes was performed. The FD scheme using fourth order formula with $n_{\xi}=41$ discretization points provided the better compromise between accuracy/efficiency.

First a multistart strategy of a SQP method (FSQP, [21]) was used to analyze the problem multimodal properties. A control discretization level $\rho=7$ was employed. Two class of interpolation polynomials for the control variable were selected: piecewise constant (PC) and piecewise linear (PL). Both cases were solved using, as initial guesses, 300 control profiles randomly generated. After analysing the multistart results two main conclusions could be extracted: first, the 
(a)

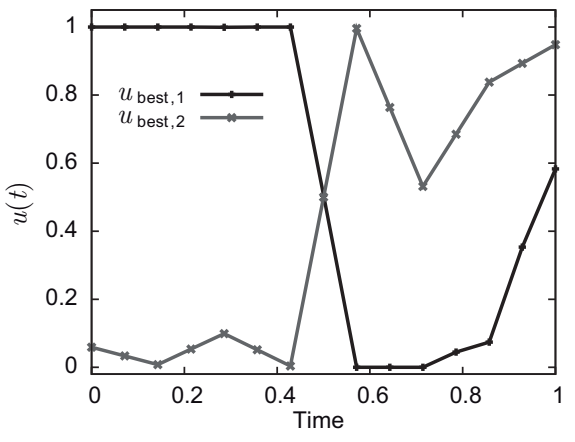

(b)

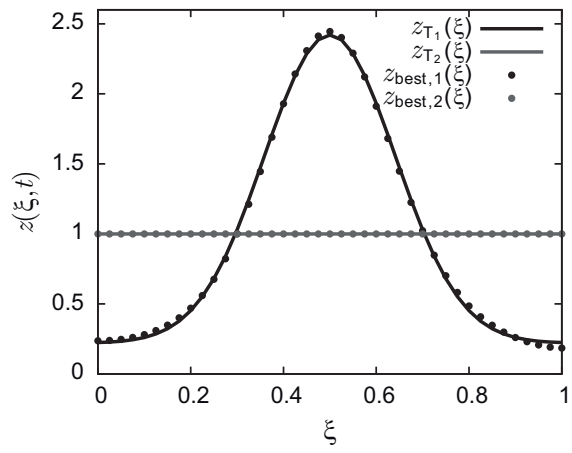

Fig. 1. (a) Optimal control profile obtained by the hybrid ( $\rho=14$, linear interpolation) technique. (b) Cell density distribution at final time.

problem is clearly multimodal and second, PL polynomials produce, as expected, better results $\left(J_{1, \text { best }}=3.58 \times 10^{-4}\right.$, $J_{2, \text { best }}=1.18 \times 10^{-8}$ ) as compared PC polynomials $\left(J_{1, \text { best }}=2.44 \times 10^{-3}, J_{2, \text { best }}=6.28 \times 10^{-6}\right)$. Thus, in the sequel the focus will be on PL polynomials. It is important to highlight that a large percentage of the optimizations end up in solutions orders of magnitude worse than the global solution. Global methods must be employed.

\section{Solution with a hybrid technique}

The metaheuristic NLP solver eSS has proved to efficiently deal with a wide range of optimization problems [6]. Therefore it has been chosen as the global NLP solver for this problem. As in the multistart approach, a discretization level $\rho=7$ with PL controls was employed. The cost functions obtained with eSS are $J_{1, \text { best }}=2.58 \times 10^{-4}$ and $J_{2, \text { best }}=$ $1.63 \times 10^{-10}$ with a computational time around 40 times lower as compared with the multistart approach. Note that the hybrid technique improves the multistart approach in both computational time and objective function value.

Solution with control refinement

The best optimal control profiles obtained in the previous step $(\rho=7)$ are now refined $(\rho=14)$. The FSQP solver is employed to compute the solution of the optimization problem. For the OCP1, the control refining resulted into $J_{1, \text { best }}=2.36 \times 10^{-4}$ in $15 \mathrm{~s}$ of extra computational effort. On the other hand, no significant improvement was found when considering OCP2. The optimal control profiles and the corresponding cell density distributions are depicted in Figure 1(a) and (b), respectively. As shown in these figures, both objectives are acquired with satisfactory accuracy.

\section{B. Case Study II: The FHN problem}

Some physiological processes, such as the heart beating, are related to electrical potential patterns. Their normal operation is associated to the formation of a traveling plane wave which spreads all over the tissue as illustrated in Figure 2(a). Under certain circumstances, such as the presence of an obstacle in the cardiac tissue, the plane front can break leading to spiral wave formation (Figure 2(b)). This class of behavior is related to neurological disorders or cardiac dysfunctions such as arrhythmia and can lead, in case the spiral breaks, to more serious problems like fibrillation. Elec- tric fields of moderate intensity can be employed to modify the behavior of this system. In this section, we propose the solution of a related dynamic optimization problem to calculate the stimulus that drives the system from the spiral behavior back to the desired one (traveling plane wave).

1) Mathematical model: The system is defined over the square spatial domain $\mathbb{V}=\left\{0 \leqslant\left(\xi_{1}, \xi_{2}\right) \leqslant 200\right\}$ with the boundary $\mathbb{B}$ being the sides of the square. The model equations are:

$$
\begin{gathered}
\frac{\partial v}{\partial t}=\left(\frac{\partial^{2} v}{\partial \xi_{1}^{2}}+\frac{\partial^{2} v}{\partial \xi_{2}^{2}}\right)+f(v, w)+u \\
\frac{\partial w}{\partial t}=g(v, w)
\end{gathered}
$$

with $f(v, w)=(\alpha-v)(v-1) v-w, g(v, w)=\varepsilon(\gamma w-$ $\delta-\beta v)$. No flux boundary conditions are considered. Initial conditions are of the form $v_{0}=1$ for $0 \leqslant \xi_{1} \leqslant 10, v_{0}=0$ for $10 \leqslant \xi_{1} \leqslant 200$ and $w_{0}=0 \forall \mathbb{V}$. Field $v$ is related to the membrane potential while $w$ collects the contributions of certain ions to the membrane current [13]. The control inputs are collected in the term $u$.

By setting the parameters $\alpha=0.1, \varepsilon=0.01, \beta=0.5, \gamma=$ 1 and $\delta=0$, the solution of system (13)- (14) is a traveling plane front as the one shown in Figure 2(a). Resetting the superior half plane at a given time instant will make the system evolve as illustrated in Figure 2(b).

The FEM with a grid of around 2300 points has been employed to solve the boundary value problem (13)-(14). Coarser grids result into low resolution front-type solutions while finer grids do not alter the solution. For optimization purposes the FEM result into a computationally involved problem. In order to overcome such limitation an accurate reduced order model derived by using the POD technique will be developed.

2) Reduced order model: In the POD, three steps can be distinguished: i) obtain a set of snapshots representative of the system behavior; ii) obtain the POD basis; and iii) project the model equations (13)-(14) over the selected POD basis. Snapshots computation: In order to obtain an accurate reduced order model, the snapshots must be representative of the system behavior. Unfortunately, there is no systematic approach to decide the conditions that better represent the 
(a)

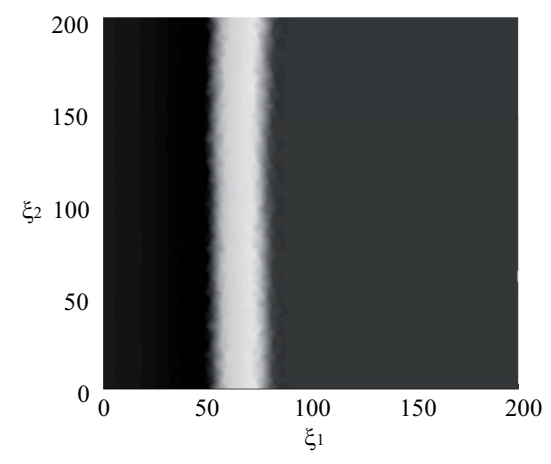

(b)

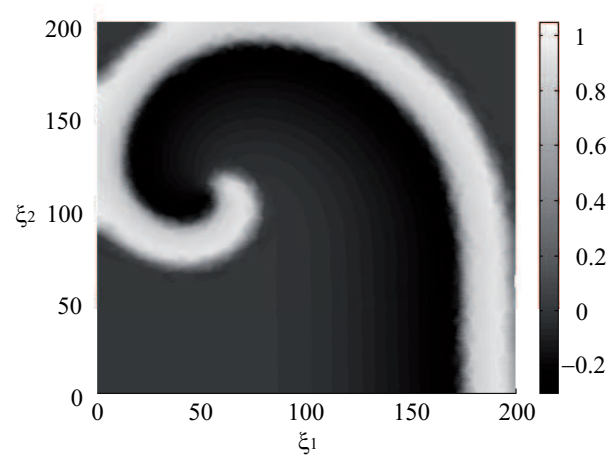

Fig. 2. Two possible solutions of FHN system corresponding to (a) front behavior and (b) spiral behavior.

system behavior. However, the idea is to capture as much information as possible from a limited set of snapshots. In our case all the snapshots were obtained from simulation of system (13)-(14). Three sets of snapshots were considered. The first two sets aimed to capture the front-type and the spiral behaviors, respectively. The last set aimed to capture the effect of the control variable. To that purpose we started from the spiral behavior and apply different control profiles. POD basis computation: Two kernels $R_{v}\left(\xi, \xi^{\prime}\right)$ and $R_{w}\left(\xi, \xi^{\prime}\right)$ will be constructed from the snapshots of the state variables $v$ and $w$, respectively, as indicated in Eqn (5). Then the POD basis are computed by solving the integral eigenvalue problem (4). As a result of this step, two basis sets $\Phi_{v}=\left[\phi_{v 1}, \phi_{v 2}, \ldots, \phi_{v n}\right]$ and $\Phi_{w}=\left[\phi_{w 1}, \phi_{w 2}, \ldots, \phi_{w m}\right]$ are obtained.

Projection of the PDE system: Projection is carried out by multiplying the original PDE system by the POD basis and integrating the result over the spatial domain $\mathbb{V}$. Note that the FEM structure may be exploited in this step [9]. In this case this procedure leads to the following ODE system:

$$
\frac{\mathrm{d} m_{v}}{\mathrm{~d} t}=P_{\mathrm{A}} m_{v}+F_{\mathrm{A}}+U_{\mathrm{A}} ; \quad \frac{\mathrm{d} m_{w}}{\mathrm{~d} t}=G_{\mathrm{A}} ;
$$

where $F_{\mathrm{A}}, G_{\mathrm{A}}$ correspond with the projection of the nonlinear terms $f(v, w)$ and $g(v, w)$, respectively. $P_{\mathrm{A}}$ and $U_{\mathrm{A}}$ result from the projection of the Laplacian operator and control terms, respectively. Different tests have been performed to find out the dimension of the basis sets $\Phi_{v}$ and $\Phi_{w}$. The best compromise between accuracy/efficiency was obtained using 85 basis in $\Phi_{v}$ and 28 in $\Phi_{w}$. The dimensionality of the resulting system is around 40 times lower as compared with the FEM. Figure 3 represents spatial distribution of the $v$ state variable at a given time instant computed using the reduced order model. Note that this solution approximates with satisfactory accuracy that one obtained using the FEM - see Figure 2(a) -.

3) Optimal control problem: The aim of this section is to design an open-loop optimal control policy $(u)$ able to drive the spiral behavior back to the plane front. For practical reasons, it is assumed that only a limited amount of actuators $\left(n_{a}=6\right)$ are available. In this regard the spatial domain is divided into six vertical bands which correspond to actuators

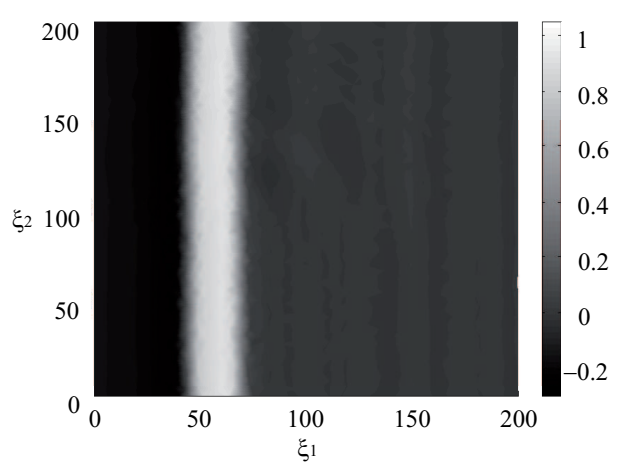

Fig. 3. ROM solution for the FHN system (front behavior).

supplying spatially independent currents. The optimal control problem is then formulated as follows: find $u_{k}(t)$ with $k=$ $1, \ldots, 6$ within $t \in[0,60]$ so as to drive the system from the spiral behavior to the desired front pattern $v_{T}\left(\xi_{1}, \xi_{2}\right)$ represented in Figure 2(a). Mathematically this is:

$$
\min _{u} J ; \quad \text { with } \quad J=\frac{1}{n_{\xi}} \sum_{i=1}^{n_{\xi}}\left(v_{i}\left(t_{f}\right)-v_{T i}\right)^{2}
$$

Subject to: i) the ROM dynamics (15) and ii) bounds on the control variables, $-1 \leqslant u_{k}(t) \leqslant 1$.

Similarly to the previous case, a multistart approach of the FSQP method was selected to study the possible multimodal nature of the problem. In this case 250 control vectors randomly chosen were considered. Each control vector was discretized into $\rho=10$ elements. Results obtained are summarized in Figure 4. A quick view to this figure shows us two things: first, the presence of several suboptimal solutions and second, the huge distance between the worst and the best solutions. Global solvers are required.

The global NLP solver eSS was employed to compute the solution of the optimization problem with $\rho=10$. The cost function value of the optimal control found was $J=$ $1.44 \times 10^{-4}$ which coincides with that of the multistart best solution although the computational time required to arrive to such a value was several orders of magnitude lower.

From that solution the FSQP method was used with a 


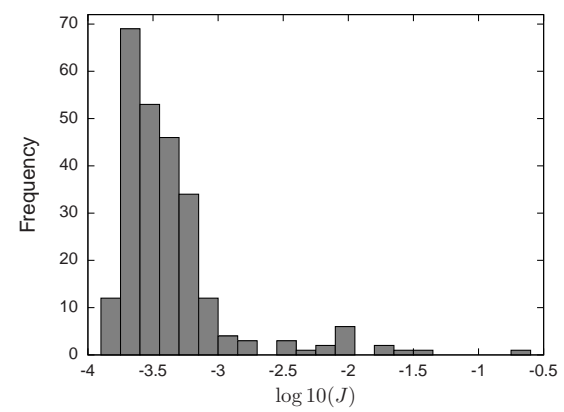

Fig. 4. Histogram of solutions for the multistart of the FHN system $\left(J_{B E S T}=1.44 \times 10^{-4}\right)$.

refining on the control discretization level $(\rho=20)$ finding a profile with $J=1.32 \times 10^{-4}$ was achieved. This optimal solution obtained using the ROM (15) was implemented in the "real" (FEM) process. The resulting $v$-field spatial distribution at final time -Figure 5- coincides with the desired one -Figure 2 (a)-.

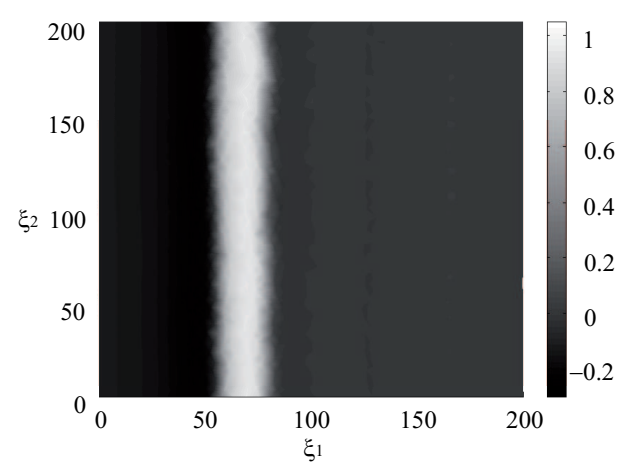

Fig. 5. $\quad v$-field final time spatial distribution after the implementation of the global optimal control profile.

\section{CONCLUSIONS AND FUTURE WORKS}

This work focused on the numerical solution of dynamic optimization problems related to spatially distributed biological systems. The difficulties related to the non-linearity and distributed nature of the processes considered were surmounted by combining different robust and efficient numerical techniques. In this regard, the control vector parametrization approach was combined with suitable hybrid global-local optimization methods and reduced order model techniques. Besides, a control refinement technique was employed to enhance efficiency.

To illustrate the usage and advantages of the proposed techniques two challenging case studies have been considered. For the first case, related to the chemotaxis process the objective was to efficiently compute the time-varying optimal concentration of chemotractant in one of the spatial boundaries in order to achieve predefined cell distribution profiles. The second case study, involving the FitzHughNagumo system, illustrates how dynamic optimization can be used to force a system to evolve from an undesired to a desired state with a reduced number of actuators.

\section{ACKNOWLEDGMENTS}

This work has been partially financed by the FP7 CAFE project KBBE-2007-1-212754), by the Spanish Ministry of Science and Innovation projects (SMART-QC, AGL2008-05267-C03-01) and (MULTISYSBIO, DPI200806880-C03-02), by CSIC intramural project (BioREDES, PIE-201170E018) and by the Xunta de Galicia project (IDECOP 08DPI007402PR).

\section{REFERENCES}

[1] A. A. Alonso, C. E. Frouzakis, and I. G. Kevrekidis. Optimal sensor placement for state reconstruction of distributed process systems. AIChE Journal, 50(7):1438-1452, 2004.

[2] E. Balsa-Canto, J. R. Banga, A. A. Alonso, and V.S. Vassiliadis. Dynamic optimization of distributed parameter systems using secondorder directional derivatives. Industrial \& Engineering Chemistry Research, 43(21):6756-6765, 2004.

[3] J. R. Banga, E. Balsa-Canto, C. G. Moles, and A. A. Alonso. Dynamic optimization of bioprocesses: Efficient and robust numerical strategies. Journal of Biotechnology, 117(4):407-419, 2005.

[4] L.T. Biegler, A.M. Cervantes, and A. Wätcher. Advances in simulaneous strategies for dynamic process optimization. Chem. Eng. Sci., 57(4):575-593, 2002.

[5] H. G. Bock and K. J. Plitt. A multiple shooting algorithm for direct solution of optimal control problems. pages 242-247, 1984.

[6] J. A. Egea, E. Vázquez, J. R Banga, and R. Martí. Improved scatter search for the global optimization of computationally expensive dynamic models. Jornal of Global Optimization, 43(2-3):175-190, 2009.

[7] R. FitzHugh. Impulses and physiological states in theoretical models of nerve membrane. Biophysical Journal, 1(6):445-466, 1961.

[8] C.A. Floudas. Deterministic Global Optimization: Theory, Methods and Applications. Kluwer Academics, The Netherlands, 2000.

[9] M. R. García, C. Vilas, J. R. Banga, and A. A. Alonso. Exponential observers for distributed tubular (bio)reactors. AIChE Journal, 54(11):2943-2956, 2008.

[10] T. Hirmajer, E. Balsa-Canto, and J.R. Banga. DOTcvpSB, a software toolbox for dynamic optimization in systems biology. BMC Bioinformatics, 10:199-213, 2009.

[11] D. Lebiedz and U. Brandt-Pollmann. Manipulation of self-aggregation patterns and waves in a reaction-diffusion system by optimal boundary control strategies. Physical Review Letters, 91(20):208-301, 2003.

[12] D. Lebiedz and H. Maurer. External optimal control of selforganisation dynamics in a chemotaxis reaction diffusion system. IEE Systems Biology, 2:222-229, 2004.

[13] J. D. Murray. Mathematical Biology I: An Introduction. SpringerVerlag, Berlin, 3rd edition, 2002.

[14] J. Nagumo, S. Arimoto, and Y. Yoshizawa. Active pulse transmission line simulating nerve axon. Proceedings of the Institute of Radio Engineers, 50(10):2061-2070, 1962.

[15] J. N. Reddy. An Introduction to the Finite Element Method. McGrawHill, 2nd edition, 1993.

[16] W. E. Schiesser. The Numerical Method of Lines. Academic Press, New York, 1991.

[17] L. Sirovich. Turbulence and the dynamics of coherent structures. Part I: Coherent structures. Quaterly of Applied Mathematics, 45(3):561$571,1987$.

[18] R. F. Stengel, R. M. Ghigliazza, and N. V. Kulkarni. Optimal enhancement of immune response. Bioinformatics, 19(9):1227-1235, 2002.

[19] V. S. Vassiliadis, C. C. Pantelides, and R. W. H. Sargent. Solution of a class of multistage dynamic optimization problems. 1. problems without path constraints. Ind. Eng. Chem. Res., 33(9):2111-2122, 1994.

[20] C. Vilas, M.R. Garcia, J.R. Banga, and A.A. Alonso. Robust feed-back control of travelling waves in a class of reaction-diffusion distributed biological systems. Physica D, 237(18):2353-2364, 2008.

[21] J. L. Zhou, A. L. Tits, and C. T. Lawrence. User's guide for FFSQP version 3.7: A fortran code for solving optimization programs, possibly minimax, with general inequality contraints and linear equality constraints, generating feasible iterates. Technical Report SRC-TR-92107r5, Institute for systems research, University of Maryland, 1997. 